\title{
5
}

\section{ATM FORUM AND ITS ACTIVITIES}

\author{
Raif O. Onvural, \\ IBM, \\ Research Triangle Park, NC 27709
}

\begin{abstract}
ATM forum is a consortium of companies world wide. Its main mission is to speed up the development and deployment of ATM products through interoperability specifications. This paper reviews the ATM forum organization and various ATM specifications produced by the membership.
\end{abstract}

\section{THE ATM FORUM ORGANIZATION}

The ATM forum was formed in October 1991. Current membership includes about 650 companies worldwide -- computer vendors, LAN and WAN vendors, switch vendors, local and long distance carriers, government and research agencies, and potential ATM users. Established originally in the US, ATM forum now includes committees in Europe and in Pacific rim.

The main mission of the ATM forum is to speed up the development and deployment of ATM products through interoperability specifications. Accordingly, the ATM forum is not a standards organization. Instead, it produces implementation agreements based on international standards, where standards are available. In other words, early deployment of ATM products requires that specifications be available much earlier than the target days of standards bodies and the goal of the ATM forum is to fill in the gaps of specifications produced by international standards.

The ATM forum can not take its specifications to any standards organizations as contributions. Instead, forum specifications have been contributed to various standards bodies by the member companies who are also members of national standards bodies. However, there is always the possibility of forum specifications to be incompatible with international standards and become a "defacto" standard.

\subsection{The Structure of the ATM forum}

Organizationally, the ATM forum has three committees governed by a board of directors, as illustrated in figure 1. The ATM forum is a non-profit mutual benefit corporation. It is managed by its board of directors with each director being elected by the members companies. Under the guidance of the board of directors, a management committee creates working groups to carry out the work of the corporation. Currently, there are three committees: global technical committee, market awareness and education committee, and enterprise network roundtable. 


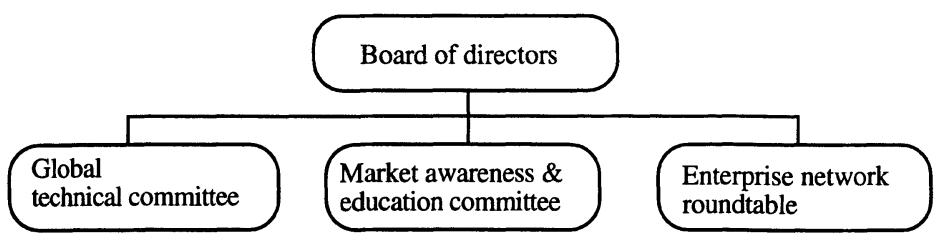

Figure 1: The ATM forum structure

Each committee has a written charter stating its purpose and objectives. Depending on their current agenda, committees may form subworking groups to tackle more specific tasks. Each subworking group has its own charter and deliverable. Participation in each subworking group activities is open to all principal forum members, with the restriction of two persons per company per subworking group.

We now proceed with a brief description of each committee.

\subsubsection{Market Awareness and Education (MA\&E) committee}

MA\&E committee was formed in August 1992. Its mission is to promote the ATM technology within both the industry and the end user community. The committee is composed of three subworking groups: end user focus group, education group, and, marketing communications and public relations group.

The main mission of the end user focus group is to design and promote end user interaction. The group undertakes market surveys to understand the requirements of end users and passes their analysis to the technical committee thereby providing input to their work.

The education group works towards raising public awareness of the ATM technology (and the ATM forum). Based on the specifications produced by the global technical committee, the group is developing presentations on ATM technology and the ATM forum specifications. The presentations are given by the forum attendees in various international conferences and trade shows.

The marketing communications and public relations group works on publicizing the efforts of the ATM forum through news releases and produces promotional items and literature.

\subsubsection{Enterprise Network Roundtable (ENR)}

ENR was formed through the efforts of MA\&E end user focus group in January 1993. The membership in ENR includes end users, customers, academia, and information systems organizations. The main mission of this committee is to provide feedback and input to the technical committee in their efforts to develop forum specifications in response to well understood and analyzed multi-industry requirements.

\subsubsection{Global Technical Committee (TC)}

All interoperability specifications are produced by the subworking groups of the technical committee. Currently, there are nine subworking groups:

- signaling

- broadband intercarrier interface 
- physical layer

- private NNI

- network management

- LAN emulation
- traffic management

- service aspects and applications

- testing

Various specifications produced by the membership is reviewed in section 2. Next, we briefly define the main objective of each group.

Broadband intercarrier interface $(\mathrm{B}-\mathrm{ICl})$ group is working towards defining a carrierto-carrier interface to provide a basic framework upon which end-to-end national and international carrier service can be facilitated. This requires the specification of various physical layer interfaces and the protocols and procedures to support the transport and multiplexing of multiple services for inter-carrier delivery.

The main focus of the physical layer group is the development of specifications for ATM transmission on different types of transmission mediums that include fiber, unshielded twisted pair, shielded twisted pair, coax, and copper.

$\mathrm{P}-\mathrm{NNI}$ group is working towards defining the private switching system to switching system interface in which a switching system may consist of a single switch (switch to switch interface) or may be a subnetwork (network to network interface).

Network management groups is focused on the specification of managed objects in ATM networks and information flows between management systems based on existing standards whenever they are available.

Service aspects and applications (SAA) is chartered to define specifications to enable new and existing applications such as audio/visual services and circuit emulation to use ATM adaptation layer (AAL) services.

Traffic management groups works on traffic aspects of ATM networking that include specifications of application traffic parameters, conformance of user traffic, development of quality of service guidelines, and definitions of service classes.

Based on specifications produced by other groups, testing group works on designing interoperability, conformance, and performance test suites.

LAN emulation group is working towards defining a LAN emulation architecture to emulate connectionless service required to support existing LAN applications without any changes over connection oriented ATM networks.

Finally, signaling group works on defining additional features, procedures, and functions to those being developed by national and international standards organizations towards addressing the signaling requirements of existing and emerging high bandwidth applications.

\subsection{ATM Forum Interfaces}

Various interfaces that have been worked on by the ATM forum TC are illustrated in figure 2.

The ATM data exchange interface (DXI) was developed to allow existing routers to interwork with ATM networks without requiring special hardware. In this specification, a DTE (a router) and a DCE (an ATM DSU) cooperates towards providing a user-network interface. DXI defines the data link protocol and physical layers that handle data transfer between a DTE and a DCE as well as local management interface and management information base for the ATM DXI.

Broadband intercarrier interface is a carrier-to-carrier interface which specifies a number of physical layer interfaces with different rates for different transmission media and supports the multiplexing of different services that include SMDS, Frame Relay, 
circuit emulation, and cell relay.

$\mathrm{NNI}$ is the interface between either two private networks or two public networks, respectively referred to as private NNI and public NNI. Private NNI is the interface between two private switching systems where a switching system can be a single switch or a subnetwork. Two main activities in this group are $\mathrm{P}-\mathrm{NNI}$ routing and $\mathrm{P}-\mathrm{NNI}$ signaling. Public NNI is being defined by ITU-TS.

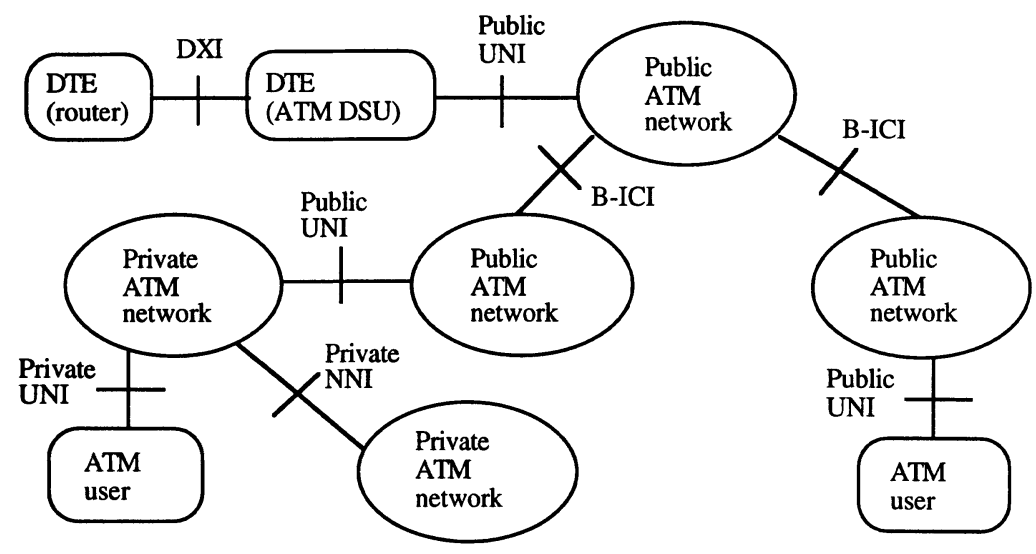

Figure 2: The ATM forum interfaces

User-network interface (UNI) is the interface between an ATM user or an end point equipment and the network. Depending on whether the network is private or public, the interface is respectively referred to as private UNI or public UNI. ATM user in this context is used to refer to a device that transmits ATM cells to the network. Accordingly, an ATM user may be an interworking unit that encapsulates data into ATM cells, a private ATM switch, or an ATM workstation. The ATM forum UNI specification include definitions of various physical interfaces and the ATM layer. For permanent virtual connections across a UNI, it defines a higher layer protocol required for UNI management. For switched virtual channels, signaling across the UNI is defined for the establishment, termination, and management of ATM connections dynamically.

\section{THE ATM FORUM IMPLEMENTATION AGREEMENTS}

The ATM forum produced its first implementation agreement in June 1992. This document entitled User-Network Interface specification version 2.0 included definitions of various physical layer interfaces and functions supporting PVCs. The second round of the ATM forum specifications were approved in the summer of 1993 and included the following implementation agreements:

- DXI specification version 1.0

- B-ICl specification version 1.0

- UNI specification version 3.0

A high level review of each document is presented next.

\subsection{UNI 3.0 specification}


Transportation of user data across an ATM network is defined in the standards as the ATM bearer service. Implementation of an ATM bearer service may be based on virtual paths (VP service) or virtual circuits (VC service), or combined virtual path and virtual circuit service (VP/NC service).

Considering application requirements, various types of connections an ATM bearer service should support include point-to-point, point-to-multipoint, and multipoint-tomultipoint connections. Furthermore, different applications have different quality of service (QoS) requirements. Hence, an ATM bearer service should be capable of supporting a variety of service metrics. In a VP service, QoS associated with the VP connection is selected to accommodate the most demanding QoS requirement of any virtual channel connection (VCC) expected to be multiplexed on to the VP. In the case of a VC service, there is a one to one correspondence between the service and the connection. Hence, the QoS requirement of the application is the QoS provided by the VC service.

Connections in ATM networks are established either dynamically (switched virtual connections, i.e. SVC) or pre-configured (permanent virtual connections, i.e., PVC).

UNI 3.0 defines standards based (to the extend it is defined and possible to use) physical and ATM layer protocols for interoperability among ATM products, i.e. ATM user and the network switch it is attached to. SVC support also requires the definition of signaling protocols, procedures, and parameters for interoperability across a UNI. Features of UNI 3.0 include specifications of the following:

- physical layer interfaces

- ATM layer

- interim local management interface

- UNI signaling

These are reviewed next.

\subsubsection{Physical Layer Interfaces}

ATM layer is independent of the physical layer. The wide availability of ATM would be benefited if various physical layer interfaces that use the existing cabling are defined for ATM. Towards this goal, ATM Forum has defined a series of interfaces including the ones listed in table 1.

\begin{tabular}{|l|c|}
\hline Transmission medium & $\begin{array}{c}\text { Total } \\
\text { transmission rate }\end{array}$ \\
\hline Multimode fiber & $100 \mathrm{Mbps}$ \\
& $155 \mathrm{Mbps}$ \\
& $155 \mathrm{Mbps}$ \\
\hline Single mode fiber & $155 \mathrm{Mbps}$ \\
\hline Shielded twisted pair & $155 \mathrm{Mbps}$ \\
\hline Coax & $44.21 \mathrm{Mbps}$ \\
\hline Unshielded twisted pair (Cat. 3) & $51 \mathrm{Mbps}$ \\
\hline Copper & $1.5 \mathrm{Mbps}$ \\
& $6.3 \mathrm{Mbps}$ \\
& $2.048 \mathrm{Mbps}$ \\
\hline
\end{tabular}

Table 1: Various ATM physical layer interfaces 
As the cabling infrastructure evolve towards all fiber, SONET/SDH is expected to be standard of choice. SDH is an international standard being developed by ITU-T and national standards bodies that include ANSI and ETSI. Developed originally by Bellcore, SDH defines a set of interfaces with speeds of multiples of $155 \mathrm{Mbps}$ (SONET starting with $51 \mathrm{Mbps}$ ). Two SDH interfaces defined by ITU-T operate at $155 \mathrm{Mbps}$ and 622 Mbps speeds.

\subsubsection{ATM layer specifications}

ATM layer transfers fixed size ATM cells to provide communication between two (or more) users of the ATM layer. This transfer occurs over an established connection. ATM layer specification in UNI 3.0 includes the following: layer

- ATM cell structure and encoding - services expected from the physical

- services provided to AAL

- traffic and congestion control

- ATM layer management specification

All these specifications except traffic and congestion control are the same as they are defined in respective ITU-TS recommendations. These are reviewed briefly next, followed by the details of traffic and congestion control specifications at the UNI.

ATM layer preserves the cell sequence integrity. ATM service data unit (SDU) is any 48 byte pattern exchanged between the ATM layer and ATM layer user. Two primitives with associated parameters used for the exchange of ATM-SDUs are defined as follows:

- ATM-DATA.request(ATM-SDU, SDU-type, submitted loss priority) congestion

- ATM-DATA.indication(ATM-SDU, SDU-type, received cell loss priority, experienced)

Similarly, ATM layer expects the physical layer to provide for the transport of ATM cells between two (or more) communicating ATM entities. Two primitives defined at the service access point (PHY-SAP) between the ATM layer and the physical layer are PHY-UNIT-DATA.request and PHY-UNIT-DATA.indication. With the former primitive, the ATM layer passes once cell and accepts one cell with the latter primitive.

ATM cell structure and encoding at the UNI is by now a quite famous figure that it is not discussed here in detail. Essentially, ATM layer supports two levels of virtual connections (VCC and VPC). The cell header also includes a 3-bit payload type indicator used to indicate whether the payload contains user data or layer management information as well as a network congestion state and network resource management information. Finally, cell loss priority bit allows the user or the network to optionally indicate the explicit cell loss priority of the cell.

The ATM layer management functions supported at the UNI include alarm surveillance for VPs and connectivity verification for VPs and VCs. These two functions are carried out by using special OAM cells. In addition to these two, ATM cells with invalid VPI/ $\mathrm{VCI}$ values are discarded and layer management is informed.

ATM networks are expected to provide service to a wide variety of applications with different QoS requirements. The QoS provided to the SVCs is agreed upon based on a traffic contract negotiated at the connection establishment phase (at subscription time for PVCs). Details of a traffic contract across a UNI is discussed later in this section.

ATM layer congestion is defined as a state of network element(s) in which the network is not able to meet the negotiated network performance objectives for connections already established in the network. ATM layer traffic control refers to the set 
of actions taken by the network to avoid possible congestion. ATM layer congestion control, then, refers to the set of actions taken by the network to minimize the intensity, spread and duration of congestion which are triggered by congestion in the network.

UNI 3.0 specification of traffic management provides definitions of various traffic parameters and a conformance checking method. The document also includes QoS requirements and guidelines as well as definitions of QoS classes for supporting different types of applications. There are four service classes defined in UNI 3.0.

- Class A: This class corresponds to constant bit rate connection oriented services with timing relation between source and destination. The two typical services of this class are $64 \mathrm{Kbps}$ voice and constant bit rate video.

- Class B: This class corresponds to variable bit rate connection oriented services with timing relation between source and destination. Variable bit rate encoded video is a typical example of this service class.

- Class C: This class corresponds to variable bit rate connection oriented services with no timing relation between source and destination. A typical services of this class is connection oriented data transfer.

- Class D: This class corresponds to variable bit rate connectionless services with no timing relation between source and destination. Connectionless data transfer between two local area networks over a wide area network is a typical example of this type of service.

In addition, user defined class of service is defined as class $X$.

-Class X: This is a raw cell service to allow proprietary AAL that may be used among terminal equipment that support the particular AAL defined by a networking vendor.

A user, at the set up phase, requests an ATM layer quality of service (QoS) from the QoS classes the network provides for ATM layer connections. Upon agreement, the network commits to meet the requested QoS as long as user complies with the traffic contract. A QoS class has either specified performance parameters (referred to as specified QoS class) or no specified performance parameters (referred to as unspecified QoS class). The former provides a quality of service to an ATM connection in terms of a subset of performance parameters defined next. In the latter case, there is no explicitly specified QoS commitment on the cell flow. This class is intended to be used for best effort service.

The QoS requirement of an application is defined through a number of parameters that may include a subset of the following:

- cell error ratio

- cell loss ratio

- cell transfer delay

- cell delay variation

- severely errored cell block ratio

- cell misinsertion rate

- mean cell transfer delay

Given this framework, a traffic contract that is agreed upon between the ATM user and the network across the UNI at the call set up phase include the following:

- requested QoS class

- conformance definition

- connection traffic descriptor

- compliant connection definition

Requested QoS class, as discussed previously, can be either specified QoS class or unclassified QoS class. If specified, then it is one of the QoS classes $1,2,3$, or 4 depending on the service class of the application, i.e. class A, B, C, or D.

Connection traffic descriptors contains a subset of source traffic descriptor, cell delay variation tolerance, conformance definition, and experimental parameters as illustrated in figure 3. 


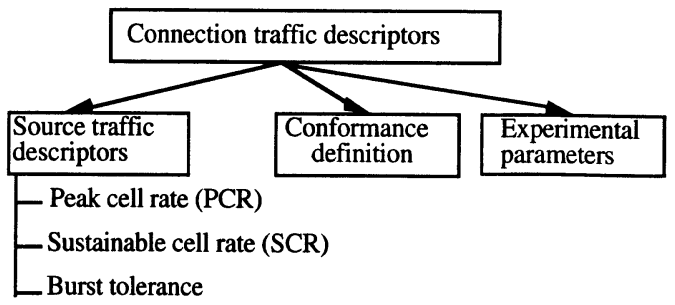

Figure 3: Connection traffic descriptors

In this context, a source traffic descriptor is a subset of traffic parameters used to capture the traffic characteristics of the connection requested by a source that specifies its various traffic aspects such as peak cell rate, sustainable cell rate, burst tolerance, and source type. In addition, experimental parameters that allow vendors to define and use additional (proprietary) metrics are allowed to be included in the signaling messages.

A generic cell rate algorithm (GCRA) is specified as the formal definition of traffic conformance. GCRA has two parameters: increment per unit time (I) and a capacity (L). In the leaky bucket version of the algorithm, the conformance of a cell is determined by using a finite capacity bucket whose contents leak out at a continuous rate of one per time unit and increase by I units per cell arrival (as long as the capacity $L$ is not exceeded). A cell that would cause the bucket to overflow is classified as nonconforming. A connection is said to be compliant as long as the number of nonconforming cells do not exceed a threshold which is defined by the network provider and specified in the traffic contract.

\begin{tabular}{|c|c|}
\hline Combination & Traffic parameters \\
\hline 1 & $\mathrm{PCR}$ for $\mathrm{CLP}=0$ and $\mathrm{PCR}$ for $\mathrm{CLP}=0+1$ \\
\hline 2 & $\begin{array}{l}\mathrm{PCR} \text { for } \mathrm{CLP}=0 \text { and } \mathrm{PCR} \text { for } \mathrm{CLP}=0+1 \\
\text { with tagging requested }\end{array}$ \\
\hline 3 & $\begin{array}{l}\text { PCR for } C L P=0+1 \text { and } S C R \text { for } C L P=0 \\
\text { BT for } C L P=0\end{array}$ \\
\hline 4 & $\begin{array}{l}\text { PCR for } C L P=0+1, S C R \text { for } C L P=0 \text { and } \\
B T \text { for } C L P=0 \text { with tagging requested }\end{array}$ \\
\hline 5 & PCR with $C L P=0+1$ \\
\hline 6 & $\begin{array}{l}\text { PCR for } C L P=0+1, S C R \text { for } C L P=0+1 \text {, } \\
\text { BT for } C L P=0+1\end{array}$ \\
\hline Best effort service & PCR for $C L P=0+1$ \\
\hline
\end{tabular}

Table 2: Allowable combinations of traffic parameters in signaling messages

When a cell is detected to be non-conforming then there are two choices: either drop the cell at the interface or allow the cell enter the network, hoping that there might be enough resources to deliver the cell to its destination. In the latter case, it is necessary to make sure that such non-conforming cells do not cause degradation to the service provided to connections that stay within their negotiated parameters. Based on this 
framework, tagging is defined as allowing non-conforming cells with cell loss priority $(C L P)=0$ enter the network after their CLP bits are changed to one. These cells are discarded first when a congestion occurs at an intermediate node so that the services provided to conforming sources will not be affected.

The allowable combinations of traffic parameters in UNI 3.0 are defined in table 2. In the case of the best effort service in which no explicit guarantees are neither required nor negotiated between the user and the network, the only traffic parameter used is $\mathrm{PCR}$ with $\mathrm{CLP}=0+1$.

\subsubsection{ATM Network Management}

UNI 3.0 interim local management interface (ILMI) provides an ATM user with the status and configuration information concerning (both VP and VC) connections available at its UNI. The ILMI communication protocol is based on the SNMP network management standard. The term interim refers to the usage of this interface until related standards are completed by the standards organizations.

The main functions provided by the ILMI include the status, configuration, and control information about the link and physical layer parameters at the UNI and address registration across UNI

Various types of information available in the ATM UNI management information base (MIB) include the physical layer, ATM layer and its statistics, VP connections, VC connections and address registration information

ILMI supports all physical layer interfaces defined by the ATM forum. It provides a set of attributes and information associated with a particular physical layer interface and status information on the state of the physical link connecting two adjacent UNI management entities (UME) at each side of the UNI.

Configuration information at the ATM layer provides information on the size of the VPI and VCl address fields that can be used by an ATM user, number of configured VPCs and VCCs, and the maximum number of connections allowed at the UNI.

VPC ILMI MIB (VCC ILMI MIB) status information indicates a UME's knowledge of the VPC (VCC) status (i.e. end-to-end, local, or unknown). Configuration information on the other hand relates to the QoS parameters for the VPC (VCC) local end point.

Address registration identifies the mechanism for the exchange of identifier and address information between an ATM user and an ATM switch port across a UNI. ATM network addresses are manually configured by the network operator into a switch port. An ATM user has its own separate identifier information (i.e., its MAC address). The end station attached to a particular switch port through the UNI would then exchange its identifier information for the ATM address information configured at the switch port through the address registration mechanism. As a result of this exchange, the end node automatically acquires the ATM network address as configured by the network operator without any requirement for the same address to have been manually provisioned into the ATM user equipment. The ATM user then appends its own identifier forming its full ATM address. Similarly, the ATM end user identifier is registered at the network and it is associated with the respective network part of the address. With this scheme, several ATM addresses with the same network defined part can be registered at the network side of the UNI.

\subsubsection{UNI Signaling}


Signaling in a communication network is the collection of procedures used to dynamically establish, maintain, and terminate connections. For each function performed, the corresponding signaling procedures define the sequence and the format of messages exchanged which are specific to the network interface across which the exchange takes place.

UNI 3.0 is built upon currently under development Q.2931 broadband signaling standard. It includes extensions to Q.2931 to support capabilities important for the early deployment and interoperability of ATM products. Three major areas of extensions are point-to-multipoint signaling, private ATM address formats, and additional traffic management capabilities.

The length of an ATM address is 20 bytes. The three private ATM addresses are defined based on the OSI network service access point (NSAP) format. An address format consists two subfields: an initial domain part (IDP) and a domain specific part (DSP). IDP specifies a subdomain of the global address space and identifies the network addressing authority responsible for assigning ATM addresses in the specified subdomain. Accordingly, IDP is further subdivided into two fields: the authority and format identifier (AFI) and the initial domain identifier (IDI). Figure 4 illustrates the high level format of an ATM address. AFI specifies the format of the IDI, the network addressing authority responsible for allocating values of the IDI, and the abstract syntax of the DSP. Three AFI's specified are Data Country Code (DCC), International Code Designator (ICD), and E164. An IDI, on the other hand, specifies the network addressing domain from which values of the DSP are allocated and the network addressing authority responsible for allocating values of the DSP from that domain.

\begin{tabular}{|l|l|l|}
\hline \multicolumn{1}{|c|}{ IDP } & \multicolumn{1}{|c|}{ DSP } \\
\hline $\begin{array}{l}\text { Authority and Format } \\
\text { Identifier (AFI) 1 octet }\end{array}$ & $\begin{array}{l}\text { Initial Domain Identifier } \\
\text { (IDI) }\end{array}$ & $\begin{array}{l}\text { Domain Specific Part } \\
\text { (DSP) }\end{array}$ \\
\hline
\end{tabular}

Figure 4: ATM address general format

UNI signaling architecture is shown in figure 5. It is a layer 3 protocol used to establish, maintain, and terminate ATM connections at the UNI. It runs on top of signaling AAL (SAAL), which defines how to transfer the signaling information for call/connection control reliably using the cells of the ATM layer on signaling virtual channels.

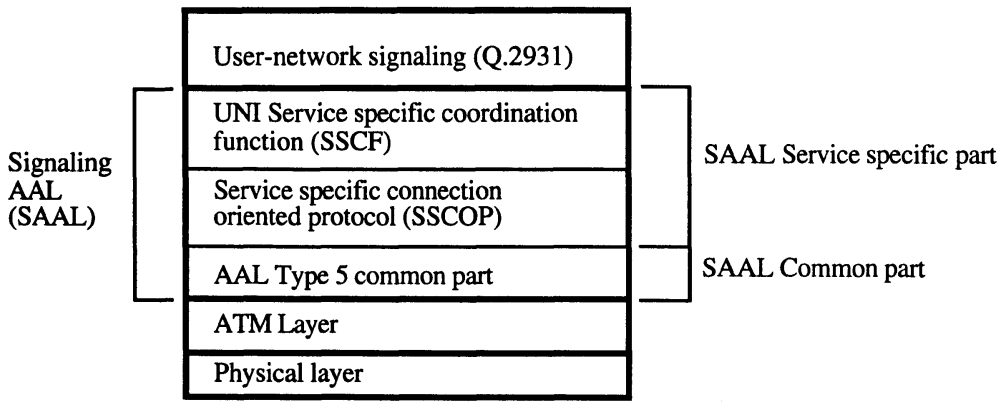

Figure 5: B-ISDN Signaling Structure 
SAAL consists of service specific and common parts. The service specific part further consists of service specific coordination function (SSCF) and service specific connection oriented protocol (SSCOP). The SAAL common part is the same as AAL type 5 common part and segmentation and reassembly sublayers.

As ATM requires connections to be established before any information can be sent, it is necessary to establish signaling channels before signaling messages can be exchanged between the two sides, i.e. the user and the network, of the UNI interface. Currently, a dedicated point-to-point signaling virtual channel with $\mathrm{VCl}=5$ and $\mathrm{VPCl}=0$ is used for all UNI signaling. The standards activities in ITU-TS are evolving towards the use of on demand signaling channels for point-to-point, point-to-multipoint, and broadcast signaling virtual channel.

\begin{tabular}{|l|l|l|l|l|l|l|l|l|}
\hline Octets & 8 & 7 & 6 & 5 & 4 & 3 & 2 & 1 \\
\hline 1 & Protocol discriminator \\
\hline 2 & 0 & 0 & 0 & 0 & Length of call reference \\
\hline 3 & flag & Call reference value \\
\hline 4 & Call reference value (continued) \\
\hline 5 & Call reference value (continued) \\
\hline 6 & Message type \\
\hline 7 & Message type (continued) \\
\hline 8 & Message length \\
\hline 9 & Message length (continued) \\
\hline etc & Variable length information elements as required \\
\hline
\end{tabular}

Figure 6: Q.2931 signaling message format

Independent of its type, the format a signaling message is defined as in figure 6. Protocol discriminator is used to distinguish Q.2931 user network control messages from those of other protocols. The length of call reference field indicates the length of the call reference in octets which identifies the call at the UNI to which the message applies. Its value is assigned by the originating side of the interface for a call and remains fixed and unique for the duration of the call. Call reference flag indicates which end of the signaling virtual channel (i.e. the network or the end point) assigned the call reference value to distinguish between incoming and outgoing messages. In addition, a global call reference value of zero is defined to be interpreted that the message received as pertaining to all call references associated with the corresponding signaling virtual channel.

\begin{tabular}{|l|l|l|l|l|l|l|l|l|}
\hline Octets & 8 & 7 & 6 & 5 & 4 & 3 & 2 & 1 \\
\hline 1 & \multicolumn{1}{|l|}{ Information element identifier } \\
\hline 2 & 1 & $\begin{array}{l}\text { coding } \\
\text { standard }\end{array}$ & IE Information field \\
\hline 3 & \multicolumn{5}{|c|}{ Length of information field } \\
\hline 4 & Length of information field (continued) \\
\hline 5, etc & Contents of the information field \\
\hline
\end{tabular}

Figure 7: General information element format

The format of an information element is given in figure 7. Each message contains a 
number of information elements which are the parameters describing some aspects of the interaction. The inclusion of information elements may be mandatory $(M)$ or optional (O). When an element is optional, notes explaining the circumstances under which such elements are included are specified in the standards documents.

We now proceed with a brief description of point-to-point and point-to-multipoint call processing in Q.2931.

A point-to-point connection is a collection of VC or VP links that connect two end points that wish to communicate to each other. As discussed previously, signaling between user and the network takes place at the edges of the network. Hence, the responsibility of finding a path between the call originator and the destination node belongs to internal network control mechanisms. The signaling messages, in this context, provide the network enough information to characterize the source and to locate the destination UNI.

The three groups of point-to-point call processing messages are defined.

i) call establishment messages such as CALL PROCEEDING, CONNECT, CONNECT ACKNOWLEDGE, and SET UP

ii) call clearing messages that include RELEASE and RELEASE COMPLETE

iii) miscellaneous messages such as RESTART, RESTART ACKNOWLEDGE, STATUS, and STATUS ENQUIRY

Next, we review the sequence of events and the messages exchanged to establish a point-to-point connection at the calling user UNI.

End systems 1 and 2 (ES1 and ES2) are connected to the ATM network across two separate UNIs. The sequence of message exchange that takes place, illustrated in figure 8, can be summarized as follows:

1. ES1 wishes to set up a connection with ES2. It sends a SETUP message across the UNI to the network node it is attached to, which contains pertinent information that identifies the two end nodes and connection characteristics.

2. The network node replies to ES1 with a CALL PROCEEDING message indicating that the SET UP message is received and connection set up is being processed. This message also includes $\mathrm{VPI} / \mathrm{VCl}$ value to be used for data traffic. Note that finding a path in the network that can meet the service requirements of the connection is an internal network function and is not part of the standards. The network sends much of the same information contained in the SET UP message sent by ÉS1 to the destination network node, which formulates a SET UP message and delivers to ES2 across the UNI ES2 is attached to the network. This SET UP message include the VPI/ $\mathrm{VCl}$ value used for data transmission.

3. If necessary, ES2 sends to network a CALL PROCEEDING message indicating that it has received the SET UP message but it will take longer to process it than the time specified.

4. ES2 decides to accept the SET UP request and forwards CONNECT message to the network node.

5. Network node sends a CONNECT ACKNOWLEDGE message to ES2. ES2 can now start sending data cells on the ATM connection using the VPI/VCl label received in the SET UP message. This message has a local significance only.

6. Network conveys the acceptance information to the network node the call is originated. Then, a CONNECT message is sent to ES1. This message contains information related to the connection set up, including VPINCI label to be used. The connection is now active in the network. 
7. ES1 processes the CONNECT message and accepts the connection set up. It sends a CONNECT ACKNOWLEDGE message to the network node. ES1 can now start sending data cells on the ATM connection using the VPI $\mathrm{VCI}$ label received in the CONNECT message.

8. Data cells flow across the UNIs in both directions.

9. ES1 wishes to terminate the connection by sending a RELEASE message across the UNI to the network.

10. The network acknowledges the receipt of RELEASE message by sending RELEASE COMPLETE message. The portion of the connection between the network and ES1 is cleared.

11. Network sends a RELEASE message to ES2 across its UNI.

12. ES2 sends RELEASE COMPLETE message to the network to acknowledge the receipt of RELEASE message. The portion of the connection between the network and ES2 is cleared.

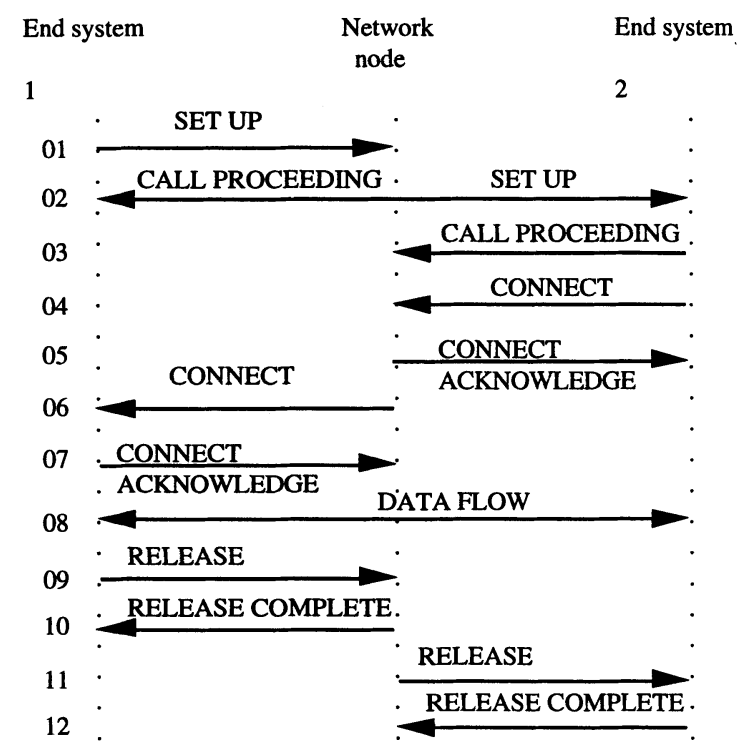

Figure 8: Principal Q.2931 flows for point-to-point ATM connections

A point-to-multipoint connection is a collection of associated VC or VP links with associated end nodes and has the following properties:

- the traffic on the connection is generated by a single node, referred as the root, and received by all other end nodes of the connection, referred to as leaf nodes (or leaves)

- there is no bandwidth reserved from the leaf nodes towards the root and that leaf nodes can not communicate with the root through the point-to-multipoint connection.

- leaf nodes can not communicate with each other directly through the point-to- 
multipoint connection.

The same VPCI/ $\mathrm{VCI}$ values are used to reach all leaf nodes and the traffic to the leaf nodes has all the same QoS, bearer capability, and ATM cell rate.

A point-to-multipoint connection is set up by first establishing a point-to-point connection between the root and a leaf node. The first SET UP message send to a leaf node has the end point reference value of zero. Furthermore, the SET UP message contains a so called broadband bearer capability information element which indicates a point-to-multipoint connection in its user plane connection configuration field. Additional leaves are added to this connection one at a time or simultaneously by the use of ADD PARTY messages. The set of messages used for point-to-multipoint signaling is given in table 3.

\begin{tabular}{|c|c|}
\hline Message Name & Definition \\
\hline ADD PARTY & add a party to an existing connection \\
\hline $\begin{array}{l}\text { ADD PARTY } \\
\text { ACKNOWLEDGE }\end{array}$ & $\begin{array}{l}\text { response to ADD PARTY message to acknowledge } \\
\text { that the add party request was successful }\end{array}$ \\
\hline $\begin{array}{l}\text { ADD PARTY } \\
\text { REJECT }\end{array}$ & $\begin{array}{l}\text { response to ADD PARTY message to acknowledge } \\
\text { that the add party request was not successful }\end{array}$ \\
\hline DROP PARTY & $\begin{array}{l}\text { clear a party from an existing connection point to } \\
\text { multipoint connection }\end{array}$ \\
\hline $\begin{array}{l}\text { DROP PARTY } \\
\text { ACKNOWLEDGE }\end{array}$ & $\begin{array}{l}\text { response to DROP PARTY message to indicate that } \\
\text { the party was dropped from the connection }\end{array}$ \\
\hline
\end{tabular}

Table 3: Point-to-multipoint signaling messages

\subsubsection{UNI 3.0 Capabilities}

In summary, various capabilities defined in UNI 3.0 signaling include the following:

- establishment of point-to-point virtual channel connections

- establishment of point-to-multipoint virtual channel connections

- three different ATM private address formats

- one ATM public address format

- symmetric and asymmetric QoS connections with declarable QoS class

- symmetric and asymmetric bandwidth connections with declarable bandwidth

- transport of network transparent parameters

- support of error handling

\subsection{Specification}

The main objective of DXI is to provide access to ATM networks for installed equipment. In particular, DXI allows a data terminal equipment (DTE), i.e. a router, and a data communications equipment (DCE), i.e. ATM-data service unit (ATM-DSU) to cooperate to provide a UNI for ATM networks. The DXI framework defines the protocols for a DTE to transport a DTE-service data unit (DTE-SDU) to a corresponding peer entity via an ATM network, as illustrated in figure 9. 


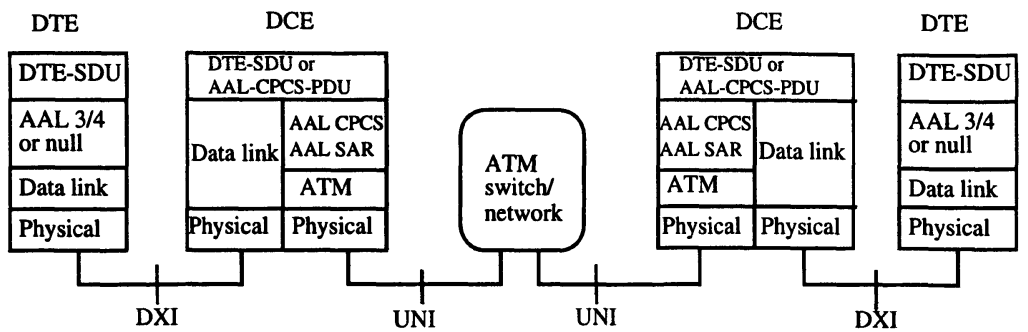

Figure 9: DXI

DXI defines a data link control protocol and physical layers which handle data transfer between a DTE and a DCE. Local management interface (LMI) and management information base (MIB) for DXI are also defined in the DXI specification.

The DTE/DCE can use V.35 or HSSI physical interfaces. DSU/ATM-switch physical layer (i.e. across UNI) can be any one of the physical layers specified in UNI 3.0.

The data link layer defines the protocol used to transport DXI frames over the physical layer between the DTE and DCE. Across a DXI, three operational modes are defined.

\subsubsection{Mode 1a}

In this mode, transport of DTE-SDU is based on AAL type 5 common part convergence (CPCS) and segmentation and reassembly (SAR) sublayers.

At the origination node, data link control layer receives DTE-SDU and encapsulates into DXI data link control frame (DXI-PDU) illustrated in figure 10. The resulting PDU is transmitted to DCE across the DXI. The DCE strips off the DXI encapsulation and obtains the values of DFA and CLP. DCE then encapsulates the DTE-SDU into AAL type 5 CPCS PDU and segments the resulting PDU into 48 byte AAL type 5 SARSDUs. DCE also maps the DFA to the appropriate VPIVCI of each cell. CLP bit value at the DXI header is also copied to the CLP bits of the transmitted cells.

\begin{tabular}{|c|c|c|c|c|c|c|c|c|c|}
\hline 01111110 & DFA & rsvd & 0 DFA & $\mathrm{CN}$ & rsvd & CLP & 11 DTE-SDU & DXI-FCS & 01111110 \\
\hline 8 bits & 6 bits & 1 & 14 bits & 1 & 1 & 1 & & 16 bits & \\
\hline
\end{tabular}

DFA: DXI frame address; CN: congestion notification; CLP: cell loss priority; rsvd: reserved; DXI-FCS: DXI frame check sequence

Figure 10: DXI data link PDU format in mode 1a

For data transmission from the ATM network to the destination DTE, the reverse process is followed. The only exception is the use of CN bit at the DXI header which is set to one if one or more cells of the DTE-DU experienced congestion in the network as specified in their cell header, EFCI bit.

\subsubsection{Mode 1b}

This mode consists of mode 1a plus transport of DTE-SDU service based on AAL 
type 3/4. When AAL type $3 / 4$ is used, DTE first encapsulates the DTE-SDU into AAL type 3/4 CPCS PDU by appending the corresponding CPCS header and trailers to the DTE-SDU. The CPCS-PDU is then encapsulated into DXI data link control frame, similar to mode 1a. The resulting frame format is illustrated in figure 11.

The resulting frame is transmitted to the DCE, which strips off the DXI header and DXI FCS thereby obtaining the DFA and CLP bit. The DCE segments the received frame into AAL type 3/4 SAR-SDUs and translates the DFA into appropriate VPI/ VCI value. CLP bit value is also copied from the DXI header to the cell header.

For data transmission from the ATM network to the destination DTE, the reverse process is followed, similar to mode 1 a.

\begin{tabular}{|c|c|c|c|c|c|c|c|c|c|c|}
\hline 01111110 & DFA & rsvd & 0 & DFA & CN & rsvd & CLP & 1 & $\begin{array}{l}\text { AAL 3/4 CPCS- } \\
\text { PDU header }\end{array}$ & DTE-SDU \\
\hline 8 bits & 6 bits 1 & 1 & 14 bits & 1 & 1 & 1 & 1 \\
\hline Flag & DXI header (2 bytes) & $<=9232$ bytes \\
\hline AAL 3/4 CPCS-PDU trailer & DXI-FCS & 01111110 \\
\hline
\end{tabular}

Figure 11: DXI data link PDU format in mode 1b with AAL type 3/4

\subsubsection{Mode 2}

In this mode, DTE operations are the same as in mode 1.b with AAL type 3/4. That is, DTE encapsulates the DTE-SDU into AAL type 3/4 CPCS PDU. The resulting PDU is then encapsulated into DXI data link control frame and it is transmitted to the DCE.

DXI header in this mode is 4 bytes long as illustrated in figure 12. Furthermore, the DXI-FCS in this mode is CRC-32 instead of CRC-16.

\begin{tabular}{|l|l|l|l|l|l|l|l|l|l|l|}
\hline 01111110 & DFA & rsvd & 0 & DFA & CN & rsvd & CLP & 1 & DFA & DFA \\
\hline Flag & 6 bits & 1 & 1 & 4 bits & 1 & 1 & 1 & 1 & 8 bits & 8 bits
\end{tabular}

Figure 12: DXI header in mode 2

At the DCE, both AAL type 5 and AAL type $3 / 4$ connections are allowed. If $A A L$ type 5 is used then DCE strips off both the DXI and AAL type 3/4 CPCS encapsulations. The remaining PDU (i.e. DXI-DSU) is then encapsulated into AAL type 5 CPCS PDU, segmented into AAL type 5 SAR-SDUs and transmitted to the ATM network using the services of ATM and physical layers, similar to the mode 1a (and 1b with AAL type 5).

If AAL type 3/4 connection is used, then DCE strips off only the DXI encapsulation and segments the AAL type 3/4 CPCS PDU into AAL type 3/4 SAR-SDUs, which are transmitted to the ATM network through ATM layer.

\subsubsection{LMI}

LMI defines the protocol for exchanging management information across DXI. It is designed to support a management station running SNMP and/or switch running UNI 3.0 ILMI protocol. 
LMI supports the exchange of various types of management information on DXI, $A A L$, and UNI.

An interested reader may refer to ATM forum ATM DXI specification version 1.0 for the details of DXI.

\section{3. $\mathrm{B}-\mathrm{ICl}$ specification}

End-to-end national and international service requires networks belonging to different carriers to be interconnected. ATM forum B-ICl facilitates this carrier-to-carrier interconnection.

B-ICl specification includes physical layer, ATM layer, and service specific functions above the ATM layer required to transport, operate, and manage a variety of intercarrier services across a B-ICl. The document also includes traffic management, network performance, and, operations and maintenance specifications. Figure 13 illustrates a generic example of carrier networks having service specific UNIs connected to other carrier ATM networks.

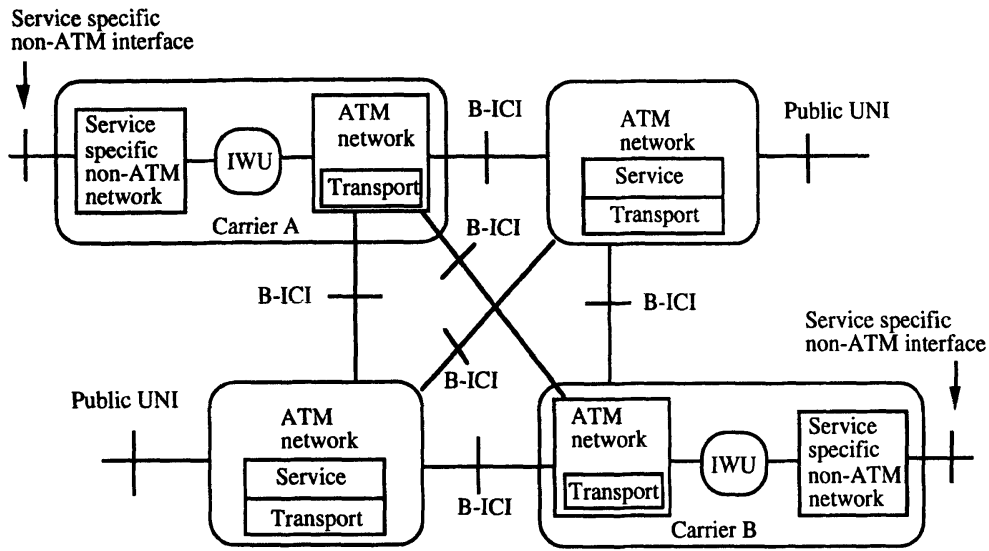

Figure 13: A generic view of service specific networks inter-connected via ATM networks

The initial $\mathrm{B}-\mathrm{ICl}$ is a multi-service interface that supports the following inter-carrier services: Cell relay service (CRS), Circuit emulation service (CES), Frame relay service (FRS) and Switched multi-megabit data service (SMDS)

The physical layer of the interface is based on ITU-TS defined network node interface (NNI) which includes SONET/SDH physical and ATM layers with the addition of DS-3 physical layer.

A service specific non-ATM network is connected to an ATM network via an interworking unit (IWU) which essentially defines an interface between the two networks. The main function performed at an IWU for each service supported across a $\mathrm{B}-\mathrm{ICl}$ is to accept ATM cells (CRS), DSn frames (CES), frame relay frames (FRS), and SMDS L3-PDU (SMDS service), and when necessary convert them into ATM cells for transmission to the ATM network. 
$\mathrm{B}-\mathrm{ICI}$ version 1.0 specification supports these services as follows:

- CRS ATM cells over PVC

- CES DSn frames encapsulated in AAL type 1 PDUs and transmitted over PVCs

- FRS frames and functions encapsulated in AAL type 5 PDUs and AAL type 5

SAR is used to form AAL type 5 SDUs. Cells are transmitted over PVCs

- SMDS interface protocol (SIP) L3 PDUs encapsulated in intercarrier service protocol connectionless service (ICIP-CLS) PDUs and AAL type 3/4 is used for transmission across an ATM network

These ATM cells are multiplexed together and passed across $\mathrm{B}-\mathrm{ICl}$ over one or more VPC and/or VCC that are pre-configured at subscription time. It is noted that this multiplexing is done at the interface level. In particular, for each service supported, there is at least one connection and cells belonging to different services are not multiplexed onto the same connection.

Traffic management and congestion control framework across $\mathrm{B}-\mathrm{ICl}$ follows very closely the framework developed in UNI 3.0. In particular, traffic parameters and connection traffic descriptor definitions in UNI 3.0 also applies to $\mathrm{B}-\mathrm{ICl}$, with some simplifications. For example, source traffic descriptor across $\mathrm{B}-\mathrm{ICl}$ is required to include service type, conformance definition, peak cell rate and cell delay variation tolerance while the inclusion of sustainable cell rate and burst tolerance in the traffic contract is an option. Network parameter control (NPC) function monitors and controls offered traffic and the validity of the ATM connection. Its main purpose is to protect network resources and the QoS of connections already established in the network during periods of congestion which might be caused by equipment malfunction or misoperation. Each carrier may use any implementation of NPC function as long as it does not violate the QoS objectives of a valid and compliant connection (or may not use any NPC function at all).

The interconnectivity across a $\mathrm{B}-\mathrm{ICl}$ is obtained by establishing a VCC and/or a VPC at the $\mathrm{B}-\mathrm{ICl}$ with a QoS suitable to meet the service requirements for end-to-end connections. B-ICl version 1.0 supports PVC only. Accordingly no call establishment or termination is associated with a PVC, i.e., Both the source and destination points of the PVC are predefined and they are fixed for the duration of the connection. The routing process provides a fixed path between two end nodes.

We will now proceed with the PVC based inter-carrier support for the four services supported across a $\mathrm{B}-\mathrm{ICl}$.

Figure 14: CRS across a $\mathrm{B}-\mathrm{ICl}$

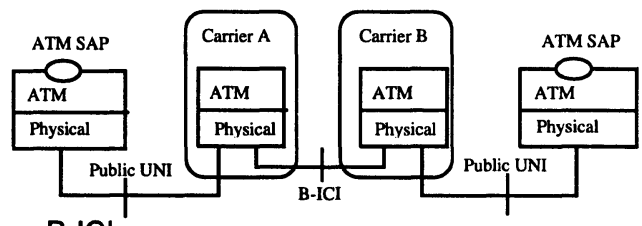

CRS is a cell based information transfer service that offers its users direct access to the ATM layer at rates upto the access link rate. Both VPC and VCC are supported. In CRS, a point-to-point PVC denotes an ATM layer VPC or VCC from a source ATM service access point (ATM-SAP) to a destination ATM-SAP, as illustrated in figure 14.

CES supports the transport of continuous bit rate (CBR) signals using ATM technology. Figure 15 shows examples of CES and its role in supporting CBR services. 
In case (a), a B-ICl supports the transport of DS $n$ signals across two public networks connecting users with UNIs at both ends. In case (b), one user is connected to an ATM network via UNI whereas the DS $n$ interface is used at the other end. Finally, in case (c), both users are connected with DS $n$ interfaces, requiring interworking functions at both ends.

(a)

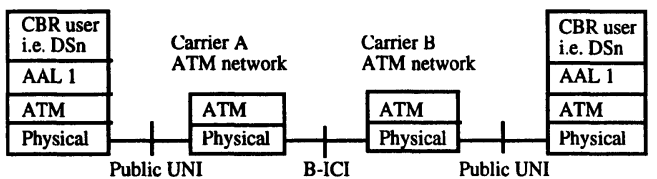

(b)

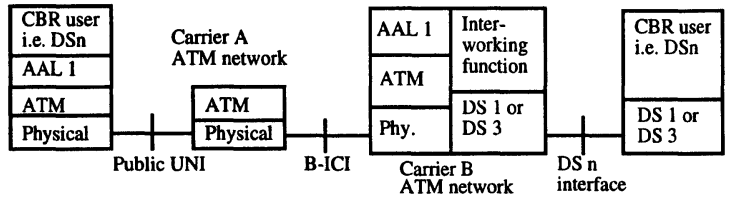

(c)

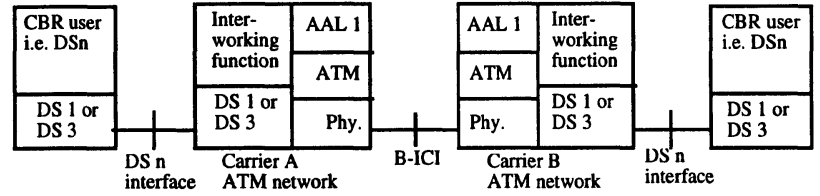

Figure 15: Circuit Emulation Service

FRS is a connection oriented data transport service. Similar to CES, two frame relay interworking scenarios are illustrated in figure 16. These are two network interworking scenarios originally defined by ITU-TS and are adopted by the forum. In the first scenario, two FR networks/CPE are connected via an ATM network whereas scenario 2 connects a FR network/CPE with a broadband CPE emulating FR.
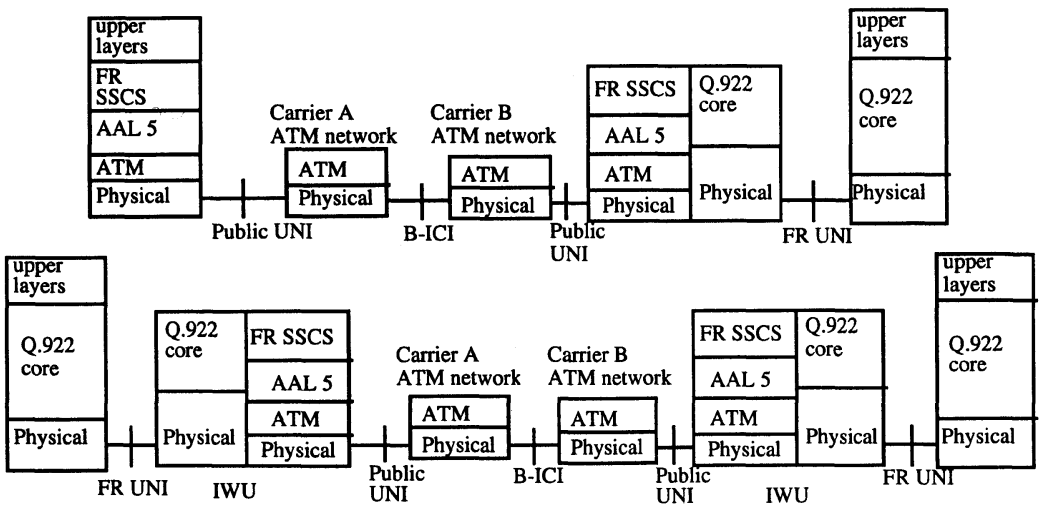

Figure 16: FES

SMDS is a public packet switched service that provides for the transport of data 
packets without the need for call establishment procedures. Customer access to the SMDS will be over the SMDS subscriber network interface (SNI). The carrier network provides an internetworking function for the encapsulation of the SMDS L3_PDU within an ICIP_CLS PDU. AAL type $3 / 4$ is used to support the transfer of these PDUs within ATM cells. Figure 17 ( MF stands for mapping function) shows an example of SMDS/ATM interworking functions.

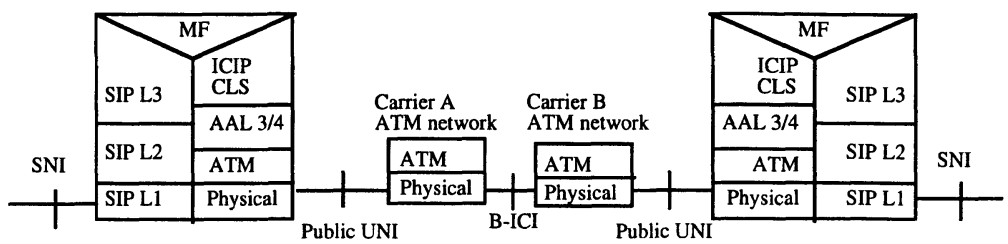

Figure 17: SMDS/ATM interworking

\section{REFERENCES}

[1] R. O. Onvural, ATM Networks: Performance Issues, Artech House Publishers, Boston, 1994

[2] ATM User-Network Interface Specification, Version 3, ATM Forum, August 1993

[3] ITU-TS Recommendation Q.2931: B-ISDN User-Network Interface Layer 3 Specification for Basic Call/Bearer Control, December 1993

[4] Bellcore TA-NWT-001111, Broadband ISDN Access Signaling Generic Requirements, Issue 1, Livingston, NJ, August 1993

[5] R. Colella, E. Gardner, and R. Collan, RFC 1237: Guidelines for OSI NSAP Allocation in Internet, Network Working Group, July 1991

[6] ITU-TS Recommendation: SSCF and SSCOP, 1993

[7] ATM DXI, Version 1.0, ATM Forum, July 1993

[8] ATM B-ICl, Version 1.0, ATM Forum, August 1993

[9] ATM Forum newsletters: 53 Bytes, 1993-1994 